\title{
Comparación de intervalos de confianza obtenidos mediante aproximación normal y bootstrap para los parámetros de la distribución Weibull
}

\section{Comparison of confidence intervals obtained by normal approximation and bootstrap for the Weibull distribution's parameters}

\author{
Luz Adriana Pereira \\ luz.pereira@correounivalle.edu.co \\ César Andrés Ojedd \\ cesar.ojeda@correounivalle.edu.co
}

\section{Resumen}

En este trabajo se analiza el desempeño de la estimación por intervalos de confianza vía aproximación normal y bootstrap paramétrico para los parámetros de la distribución Weibull en dieciocho escenarios simulados. Bajo diferentes tamaños de muestra, se valora el efecto del parámetro de forma $\beta$ en la estimación por intervalos para el parámetro de escala $\eta$. De igual manera, se varía el parámetro de escala en la estimación por intervalos para el parámetro de forma. Se encuentra que los intervalos de confianza construidos bajo aproximación bootstrap presentan un mejor comportamiento en comparación con los obtenidos por aproximación normal en cuanto a la probabilidad de cobertura y longitud mediana. Los intervalos de confianza al $(1-\alpha) \times 100 \%$ por aproximación normal para $\beta$ resultaron asimétricos con respecto al error $\alpha$. En ambos métodos de estimación se evidencia que para $\beta \geq 2$, los intervalos de confianza para el parámetro $\eta$ disminuyen la longitud.

Palabras clave: distribución de valores extremos mínimos, distribución Weibull, intervalo de confianza, probabilidad de cobertura..

\begin{abstract}
In this paper we analyze the performance of the confidence intervals estimation via normal approximation and parametric bootstrap for the parameters of the Weibull distribution in eighteen simulated scenarios. Under different sample sizes, the effect of the form parameter $\beta$ on the interval estimate is evaluated for the scale parameter $\eta$. In the same way, the scale parameter is varied in the interval estimation for the shape parameter. We found that the confidence intervals constructed
\end{abstract}

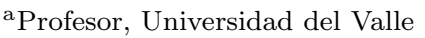

${ }^{\mathrm{b}}$ Profesor, Universidad del Valle
} 
under the bootstrap approach show a better behavior in comparison with those obtained by normal approximation in terms of coverage probability and median length. The confidence intervals at $(1-\alpha) \times 100 \%$ by normal approximation for $\beta$ were asymmetric with respect to the error $\alpha$. In both methods, it is evident that for $\beta \geq 2$ the confidence intervals for the parameter $\eta$ decrease the length.

Keywords: confidence interval, coverage probability, smallest extreme values distribution, Weibull distribution..

\section{Introducción}

Dada la gran popularidad del uso de la distribución Weibull, debido a la variedad de formas que le es posible ajustar, numerosos trabajos técnicos se encuentran disponibles en la literatura relacionados con la estimación por intervalos de confianza para los parámetros de esta distribución; sin embargo, son escasos aquellos trabajos en los que se presente de manera sencilla por medio de indicadores las debilidades o ventajas de los métodos de estimación por intervalos y, por consiguiente, los cuidados que se debe tener al realizar inferencias sobre los parámetros de la distribución Weibull.

(Ostrouchov \& Meeker 1988) concluyen que los intervalos de confianza basados en el test de la inversión del logaritmo de la verosimilitud presentan un mejor desempeño que los intervalos vía aproximación normal, lo anterior en presencia de censura tipo I para la distribución Weibull y la lognormal. (Vander Weil \& Meeker 1990) analizan intervalos de confianza en presencia de datos con censura tipo I que provienen de pruebas aceleradas y encuentran que los intervalos de confianza basados en la razón de verosimilitud tienen probabilidades de cobertura más cercanas a la confianza nominal en comparación con el procedimiento por aproximación normal. (Doganaksoy 1995) revisa la utilidad práctica de los intervalos vía logaritmo de razón de verosimilitud en situaciones reales de datos de tiempo de vida y concluye que son pocas sus aplicaciones debido a la dificultad computacional de su implementación. (Meeker \& Escobar 1998) presentan la estimación por intervalos de confianza para los parámetros de la distribución Weibull vía intervalos de confianza para los parámetros de la distribución de valores extremos mínimos.

Es bien conocido que la distribución Weibull es comúnmente utilizada en estudios de confiabilidad y así, numerosos usuarios (no todos con una formación rigurosa en estadística) se enfrentan a tomar decisiones con base en inferencias realizadas a partir de un conjunto de datos, los cuales se asume que provienen de un modelo probabilístico Weibull. En este proceso, es inevitable que los usuarios se enfrenten a las siguientes situaciones: (a) las ecuaciones para los estimadores de máxima verosimilitud no son expresiones cerradas, (b) los estimadores obtenidos en estos métodos son sesgados y la cantidad del sesgo es desconocida, (c) la distribución Weibull cumple con las condiciones de regularidad solo cuando el parámetro de forma es mayor a 2, puesto que en otros casos se obtienen estimadores de máxima 
verosimilitud altamente variables de una muestra a otra (Cousineau 2009). Adicionalmente, se enfrentan a la incertidumbre de cómo la estimación, por ejemplo, del parámetro de forma influye en la estimación del parámetro de escala, y viceversa. De manera que este trabajo tiene como propósito presentar, a través de diferentes escenarios de simulación, una respuesta a quienes desean realizar inferencias sobre los parámetros de la Weibull por medio de intervalos de confianza, ¿cuál es el comportamiento de los intervalos de confianza en esta distribución?, ¿son sesgados?, ¿en qué escenarios es recomendable realizar estimación por aproximación a la distribución normal? Para ello, se propone comparar la longitud mediana y la probabilidad de cobertura de intervalos de confianza construidos vía aproximación normal versus bootstrap paramétrico, construir indicadores del desempeño de los intervalos de confianza que permitan concluir en qué situaciones resulta adecuado cada método de estimación propuesto e incluso a través de gráficas ilustrar el comportamiento de los intervalos de confianza, siendo posible observar el efecto del tamaño de muestra, la asimetría y la variabilidad de las estimaciones.

Según (Meeker \& Escobar 1998), aunque los intervalos construidos con base en la aproximación normal sean sencillos de calcular, tales aproximaciones pueden resultar pobres en desempeño. Una importante deficiencia en el desempeño de los intervalos de confianza obtenidos por aproximación normal está relacionada con la amplia diferencia entre la probabilidad de cobertura y el nivel de confianza nominal excepto cuando el número de fallas observadas es grande.

La metodología aplicada en este trabajo para valorar el comportamiento de los intervalos de confianza en el caso de la distribución Weibull se desarrolla con base en simulaciones. En la segunda sección se define el modelo de probabilidad Weibull, algunas propiedades, su relación con la distribución de valores extremos mínimos y los escenarios de simulación considerados. En la tercera sección se presentan los resultados encontrados en las simulaciones junto con una aplicación a datos de tiempos de sobrevivencia en pacientes con cáncer avanzado de pulmón. Finalmente, se presentan las conclusiones.

\section{Modelo de probabilidad y escenarios de simula- ción}

Sea $T$ una variable aleatoria con soporte en los reales positivos. Si $T$ sigue un modelo paramétrico Weibull con parámetros de escala $\eta>0$ y de forma $\beta>0$, entonces $T \sim$ weibull $(\eta, \beta)$ y su función de distribución acumulada es

$$
\operatorname{Pr}(T \leq t ; \eta, \beta)=1-\exp \left[-\left(\frac{t}{\eta}\right)^{\beta}\right], \quad t>0 .
$$

La media de $T$ está dada por $\mathrm{E}(T)=\eta \Gamma\left(1+\frac{1}{\beta}\right)$ y la varianza por $\operatorname{Var}(T)=$ $\eta^{2}\left[\Gamma\left(1+\frac{1}{\beta}\right)-\Gamma^{2}\left(1+\frac{1}{\beta}\right)\right]$. Por otro lado, el $p$-cuantil para la distribución Weibull está definido como $t_{p}=\eta[-\log (1-p)]^{\frac{1}{\beta}}$. 
El parámetro $\eta$ en la distribución Weibull coincide con el cuantil 0.632. En confiabilidad, resulta importante la estimación del parámetro $\beta$, ya que permite caracterizar la tasa de falla asociada a un componente. Para $0<\beta<1$ la tasa de falla es decreciente, para $\beta>1$ la tasa de falla es creciente y $\beta=1$ es constante (Meeker \& Escobar 1998).

Los escenarios de simulación planteados en este trabajo involucran diferentes tamaños de muestra $n=\{10,20,50,100\}$ junto con diferentes combinaciones de los parámetros de forma $\beta=\{0.5,1,2\}$ y de escala $\eta=\{0.5,1,2\}$. Los intervalos fueron construidos a un nivel de confianza del $(1-\alpha) \times 100 \%$ con $\alpha=0.05$.

Un aspecto importante por definir en el momento de llevar a cabo un estudio vía simulación es el número de simulaciones que serán necesarias para encontrar resultados que minimicen la variabilidad de las estimaciones. Siguiendo la metodología propuesta por (Koehler et al. 2009), se encontró que aproximadamente de 10,000 simulaciones, tanto para el parámetro de forma como para el de escala, no se evidencian marcadas diferencias en la estimación de la probabilidad de cobertura alcanzada por los intervalos vía aproximación normal. De igual manera, la variabilidad del porcentaje de sesgo se estabiliza alrededor de esta cantidad de simulaciones. En este estudio se realizaron 10,000 estimaciones por intervalos de confianza vía aproximación normal y bootstrap paramétrico.

Inicialmente, se define la distribución de valores extremos mínimos (SEV, por sus iniciales en idioma inglés Smallest Extreme Values). La variable aleatoria, $-\infty<Y<\infty$, se distribuye $\operatorname{SEV}$, es decir $Y \sim \operatorname{SEV}(\mu, \sigma)$, si su función de densidad de probabilidad existe y está dada por (Meeker \& Escobar 1998):

$$
f(y ; \mu, \sigma)=\frac{1}{\sigma} \phi_{\mathrm{SEV}}\left(\frac{y-\mu}{\sigma}\right) \quad-\infty<y<\infty
$$

En la ecuación (2), $\phi_{S e v}(z)=\exp [z-\exp (z)]$ es la función de densidad de probabilidad para la distribución SEV estandarizada $(\mu=0, \sigma=1),-\infty<\mu<\infty$ es el parámetro de localización y $\sigma>0$ es el parámetro de escala. Según (Coles 2001) la distribución SEV cumple con las condiciones de regularidad, de tal manera que los estimadores $\hat{\mu}$ y $\hat{\sigma}$ obtenidos por máxima verosimilitud son eficientes, óptimos, asintóticamente normales, consistentes e invariantes ante transformaciones.

Según (Meeker \& Escobar 1998), usando las estimaciones máximo verosímiles de los parámetros de la distribución SEV y la matriz de varianzas-covarianzas de estas estimaciones, se construyen intervalos de confianza para $\mu$ y $\sigma$, y a partir de estos intervalos, bajo transformaciones adecuadas de sus límites, es posible obtener una estimación por intervalo de los parámetros de la distribución Weibull. Dado que la distribución SEV cumple con las condiciones de regularidad, es posible garantizar la asintoticidad normal de las estimaciones máximo verosímiles de sus parámetros. De esta forma, es adecuado aproximar la distribución de la estimación del parámetro de localización $\mu$ por medio de $Z_{\hat{\mu}}=\frac{\hat{\mu}-\mu}{\sec _{\hat{\mu}}}$ a una distribución $\mathrm{N}(0,1)$ y obtener un intervalo de confianza al $(1-\alpha) \times 100 \%$ para $\eta$ bajo la siguiente 
expresión:

$$
\langle\eta\rangle_{1-\alpha}=\left[\exp \left(\hat{\mu}-\Phi^{-1}(1-\alpha) \times \hat{s} e_{\hat{\mu}}\right) ; \exp \left(\hat{\mu}+\Phi^{-1}(1-\alpha) \times \hat{s} e_{\hat{\mu}}\right)\right]
$$

Donde $\Phi^{-1}(1-\alpha)$ corresponde al cuantil $1-\alpha$ en la distribución normal estándar.

Por otro lado, al aproximar la distribución muestral del estimador $\log (\hat{\sigma})$ por medio de $Z_{\log (\hat{\sigma})}=\frac{(\log (\hat{\sigma})-\log (\sigma))}{\hat{s} \log _{\log }(\hat{\sigma})}$ a una distribución normal estándar, se obtiene un intervalo de confianza al $(1-\alpha) \times 100 \%$ para $\beta$,

$$
\langle\beta\rangle_{1-\alpha}=\left[\frac{1}{\hat{\sigma} \times \exp \left(\Phi^{-1}(1-\alpha) \times \frac{\hat{s} \hat{\sigma}}{\hat{\sigma}}\right)}, \frac{1}{\frac{\hat{\sigma}}{\exp \left(\Phi^{-1}(1-\alpha) \times \frac{s e_{\hat{\sigma}}}{\hat{\sigma}}\right)}}\right]
$$

Según (Meeker \& Escobar 1998), cuando el tamaño de muestra es pequeño, este método presenta deficiencias tales como que la probabilidad de cobertura sea significativamente menor a la probabilidad nominal especificada y que la longitud de los intervalos generados pueden resultar ser cero o incluso obtenerse longitudes muy amplias. (Jeng \& Meeker 2000), mencionan que la idea de emplear el remuestreo bootstrap para construir intervalos de confianza para los cuantiles resuelve los inconvenientes que se presentan en los intervalos de confianza estimados bajo aproximación normal en muestras con censura tipo I en una distribución Weibull, al reducir la necesidad de muestras grandes para alcanzar rápidamente las aproximaciones a la normalidad en la distribución de los estimadores puntuales. (Efron 1985), a través de la técnica del bootstrap, propuso métodos para la construcción de intervalos de confianza aproximados en un parámetro $\theta$ bajo la distribución de la estadística $\hat{\theta}$. Un intervalo de confianza al $(1-\alpha) \times 100 \%$ para $\theta$ basado en el supuesto que la distribución simulada de $Z_{\hat{\theta}^{*}}$ suministra una buena aproximación de la distribución de $Z_{\hat{\theta}}$ es:

$$
\langle\theta\rangle_{1-\alpha}=\left[\hat{\theta}-Z_{\hat{\theta}_{1-\frac{\alpha}{2}}^{*}} \hat{s e} \hat{\theta}, \hat{\theta}-Z_{\hat{\theta}_{\frac{\alpha}{2}}^{*}} \hat{s e} e_{\hat{\theta}}\right]
$$

Donde $Z_{\hat{\theta}_{1-\frac{\alpha}{2}}^{*}}$ y $Z_{\hat{\theta}_{\frac{\alpha}{2}}^{*}}$ son los cuantiles $1-\frac{\alpha}{2}$ y $\frac{\alpha}{2}$ de la distribución de $Z_{\hat{\theta}^{*}} . \hat{\theta}$ junto con $\hat{s e}_{\hat{\theta}}$ son estimaciones provenientes de la muestra de partida para el bootstrap.

Dos conceptos importantes que se deben considerar al evaluar los intervalos de confianza son la longitud del intervalo y la probabilidad de cobertura. Estos criterios no se pueden analizar por separado porque no es deseable un intervalo con probabilidad de cobertura alta si su longitud es muy grande o un intervalo con una longitud muy pequeña pero con probabilidad de cobertura muy baja. En este trabajo fue calculada la probabilidad de cobertura y la longitud mediana para los intervalos obtenidos por aproximación normal y bootstrap paramétrico. La probabilidad de cobertura es calculada como el cociente entre el número de intervalos simulados que contienen el valor del parámetro y el total de intervalos simulados. La longitud mediana corresponde al percentil 50 de la distribución de la longitud 
de los intervalos simulados. Con base en estas dos medidas se construyeron dos indicadores del desempeño de los intervalos de confianza, el $\mathrm{R}_{\mathrm{LMI}}$ y el $\mathrm{R}_{\mathrm{PC}}$, los cuales corresponden a razones entre las probabilidades de cobertura y longitudes medianas encontradas entre los métodos de estimación. La ecuación (4) corresponde a la expresión del $R_{\mathrm{LMI}}$ y la ecuación (5) a $\mathrm{R}_{\mathrm{PC}}$ :

$$
\mathrm{R}_{\mathrm{LMI}}=\frac{\mathrm{LMI}_{\mathrm{AN}}}{\mathrm{LMI}_{\mathrm{BP}}}
$$

En la ecuación (4), $\mathrm{LMI}_{\mathrm{AN}} \mathrm{y} \mathrm{LMI}_{\mathrm{BP}}$ hacen referencia a la mediana de la longitud de los intervalos de confianza obtenidos por aproximación normal (AN) y bootstrap paramétrico $(\mathrm{BP})$, respectivamente. Este indicador es de fácil interpretación: si $\mathrm{R}_{\mathrm{LMI}}<1$ entonces el método $\mathrm{AN}$ arroja longitudes medianas más pequeñas en comparación con el método BP. Por el contrario, si $\mathrm{R}_{\mathrm{LMI}}>1$ el método de estimación BP arroja intervalos de confianza $50 \%$ de las veces con longitudes más pequeñas que el método $\mathrm{AN}$.

$$
\mathrm{R}_{\mathrm{PC}}=\frac{\mathrm{PC}_{\mathrm{AN}}}{\mathrm{PC}_{\mathrm{BP}}}
$$

La ecuación (5) hace referencia a la razón entre la probabilidad de cobertura de los intervalos de confianza obtenidos por aproximación normal y por bootstrap paramétrico, denotado por $\mathrm{PC}_{\mathrm{AN}}$ y $\mathrm{PC}_{\mathrm{BP}}$, respectivamente. Al igual que el indicador propuesto en la ecuación (4), $\mathrm{R}_{\mathrm{PC}}$ es fácilmente interpretable: si $\mathrm{R}_{\mathrm{PC}}<1$, entonces el método AN arroja probabilidades de cobertura más pequeñas en comparación con el método BP.

\section{Resultados y discusión}

Los resultados presentados en esta sección fueron obtenidos a través de 10,000 simulaciones realizadas en el paquete estadístico R (Team 2017). Para la obtención de las estimaciones de los parámetros por máximo verosimilitud de la distribución de valores extremos mínimos se hizo uso de la función gum.fit de la librería ismev desarrollada por (Heffernan \& Stephenson 2016).

Los resultados de la Tabla 1 evidencian que no hay efectos del parámetro $\eta$ en la longitud del intervalo de confianza para el parámetro de forma $\beta$, ya que no se observan cambios en los valores frente la mediana de las longitudes tanto en los intervalos obtenidos por aproximación normal como por bootstrap paramétrico. Con respecto a la mediana de las longitudes obtenidas en cada uno de los métodos de estimación por intervalos, no se observan marcadas diferencias, razón por la cual el indicador $\mathrm{R}_{\mathrm{Lm}}$ presenta valores muy cercanos a 1 . En cuanto al otro aspecto importante a valorar, tal como lo es la probabilidad de cobertura, se encuentra que el método de estimación por aproximación normal requiere de los tamaños de muestra más grandes considerados en el estudio, $n=50$ o $n=100$, para acercarse 
al nivel de confianza nominal del $95 \%$. Caso contrario ocurre con el método de estimación bootstrap, puesto que se acerca rápidamente la probabilidad de cobertura a la confianza nominal del $95 \%$ e incluso en algunas ocasiones la supera, sin que se vea afectado la longitud del intervalo. El indicador $\mathrm{R}_{\mathrm{PC}}$ refleja esta potencialidad del método bootstrap al encontrarse alrededor de valores cercanos a 0.98. El indicador $\mathrm{R}_{\mathrm{LMI}}$ toma un valor en promedio de 1.02 y una desviación estándar de 0.04, lo que sugiere poca variabilidad entre las razones de las longitudes medianas encontradas en cada uno de los métodos bajo los escenarios simulados y, además, que en la mayoría de los casos los intervalos de confianza estimados por aproximación normal y bootstrap no mostraron diferencias marcadas en las longitudes de los intervalos estimados. De igual forma, el indicador $\mathrm{R}_{\mathrm{PC}}$ toma un valor en promedio de 0.98 y una desviación estándar de 0.02 , lo que sugiere que el método de estimación por aproximación normal en ocasiones arrojó intervalos de confianza para los cuales su probabilidad de cobertura no alcanzó la confianza nominal.

La Tabla 2, presenta la longitud mediana y la probabilidad de cobertura alcanzadas en la estimación por intervalos para el parámetro de escala $\eta$, valorando el efecto del parámetro de forma $\beta$. Los resultados muestran que las longitudes medianas encontradas, para los intervalos de confianza en ambos métodos se ven afectadas al cambiar el valor del parámetro de forma, ya que en los escenarios simulados se encontró que entre más simétrica es la distribución, es decir, cuando $\beta \geq 2$, la longitud de los intervalos disminuye. El indicador $\mathrm{R}_{\mathrm{LMI}}$ fue en promedio 0.91 con una desviación estándar de 0.08 , lo que significa que en la mayoría de los escenarios simulados el método de aproximación normal entrega estimación por intervalos de menor longitud que el método bootstrap paramétrico, aunque cuando se tienen muestras grandes, en el caso de $n=100$, no se observan diferencias en las longitudes. Con respecto a las probabilidades de cobertura, el método de estimación por intervalos basado en aproximación normal en pocos escenarios de los simulados alcanzó el nivel de confianza nominal a diferencia de lo que ocurrió con el método basado en bootstrap paramétrico, en el cual para escenarios de tamaño de muestra pequeños considerados, $n=10$ o $n=20$, la probabilidad de cobertura alcanzó la confianza nominal del $95 \%$. El valor promedio del indicador $\mathrm{R}_{\mathrm{PC}}$ es igual a 0.98 con un 0.02 de desviación estándar, evidenciando que el método bootstrap entrega mejores probabilidades de cobertura.

La Figura 1 y 2 ilustra el comportamiento de las estimaciones del límite superior e inferior para 200 intervalos de confianza construidos al $95 \%$ bajo aproximación normal y bootstrap, respectivamente, para el parámetro de forma $\beta=1$. Se consideró en este caso un parámetro de escala $\eta=0.5$ y se observó bajo diferentes tamaños de muestra que los intervalos de confianza estimados por aproximación normal son asimétricos con respecto al error $\alpha$, ya que la estimación del límite inferior del intervalo tiende a estar más cerca del valor esperado de la estimación del parámetro y resulta ser menos variable que la estimación obtenida para el límite superior. Cabe destacar que a medida que se incrementó el tamaño de la muestra, esta asimetría se fue corrigiendo. De otro lado, el método bootstrap no presenta este comportamiento asimétrico. Este comportamiento se presenta en los nueve escenarios de simulación resultantes de variar el parámetro $\eta$ y el tamaño 
de muestra $n$.

Respecto al comportamiento de las estimaciones de los límites de los intervalos de confianza para el parámetro de escala $\eta$, las Figuras 3 y 4 , ilustran el comportamiento de estas estimaciones y no se hizo evidente asimetría en las estimaciones con respecto al error $\alpha$ en ambos métodos. En este caso, el parámetro de forma de la Weibull toma el valor de $\beta=1$ y el de escala $\eta=0.5$.

\subsection{Un ejemplo: tiempos de sobrevivencia en pacientes con cáncer de pulmón}

En esta sección se ilustra el procedimiento de estimación por intervalos de confianza considerando el conjunto de datos lung de la librería survival (Therneau 2015, Therneau \& Grambsch 2000) que contiene información de pacientes con cáncer avanzado de pulmón obtenidos por el North Central Cancer Treatment Group. De las variables disponibles, se analiza el tiempo de sobrevivencia en días registrado para 227 pacientes. Con el propósito de que el lector pueda replicar los resultados obtenidos se presentan los códigos utilizados en el proceso.

La Figura 5 se construye para valorar la bondad de ajuste de los tiempos de sobreviviencia a la distribución Weibull. El gráfico cuantil-cuantil (qqPlot, (Roth 2016)) sugiere que la distribución Weibull es un modelo razonable para estos datos.

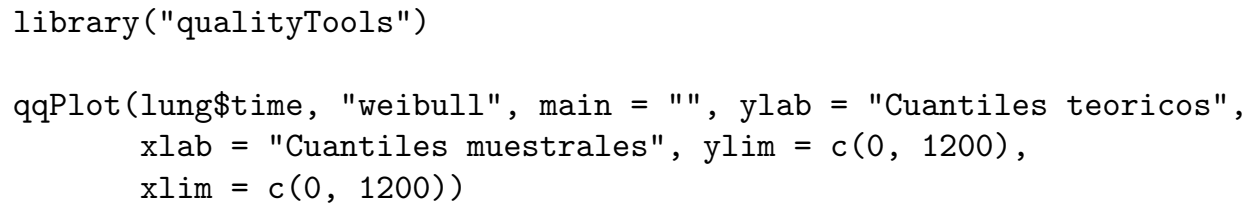

Una vez definido la distribución Weibull como un modelo probabilístico para este conjunto de observaciones, se realiza una transformación logarítmica a los datos con el fin de obtener por medio de esta transformación una variable aleatoria con distribución SEV y proceder a la estimación de los parametros por máxima verosimilitud. Haciendo uso de la función gum.fit del paquete ismev y realizando las consideraciones necesarias en la función para realizar la estimación por máxima verosimilitud para la distribución de valores extremos mínimos se obtiene:

ajus.sev <- gum.fit $($ xdat $=-\log ($ lung\$time $)$, show $=$ FALSE $)$

ajus.sev\$mle*c $(-1,1)$

[1] $5.8192528 \quad 0.6817189$

ajus.sev\$se

[1] 0.047484550 .03517363

De esta manera se obtienen las estimaciones MLE para $\mu$ y $\sigma$ en la distribución 
SEV. Finalmente, es posible construir una estimación por intervalos para $\eta$ y $\beta$ al $95 \%$ de confianza vía aproximación normal, bajo las siguientes expresiones:

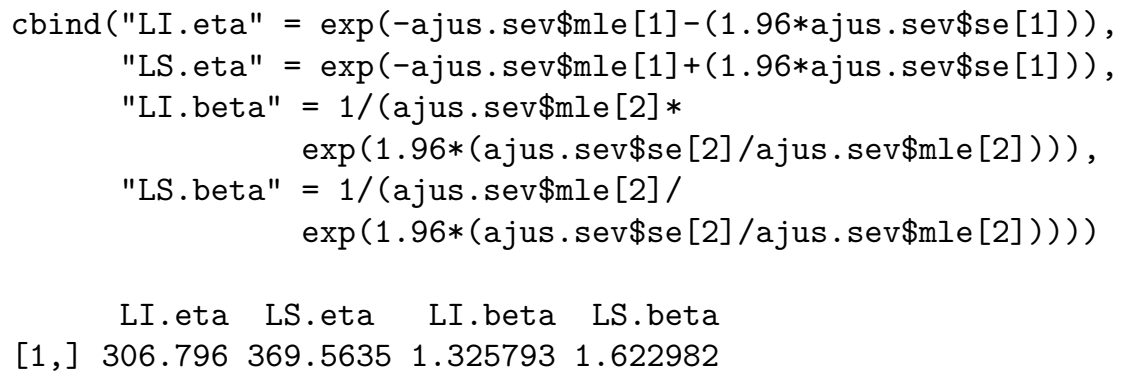

En la estimación por intervalos vía bootstrap paramétrico, una vez realizada la estimación por máxima verosimilitud para $\mu$ y $\sigma$, estas estimaciones iniciales son utilizadas para generar 2,000 muestras bootstrap con distribución

Weibull $\left(\eta=\exp (\mu), \beta=\frac{1}{\sigma}\right)$. Luego, en cada muestra se realiza la estimación MLE y se procede a construir los intervalos de confianza. El siguiente código se utiliza para la estimación por intervalos bootstrap. Con el objetivo de optimizar la función se hace uso de la librería snowfall (Knaus 2015).

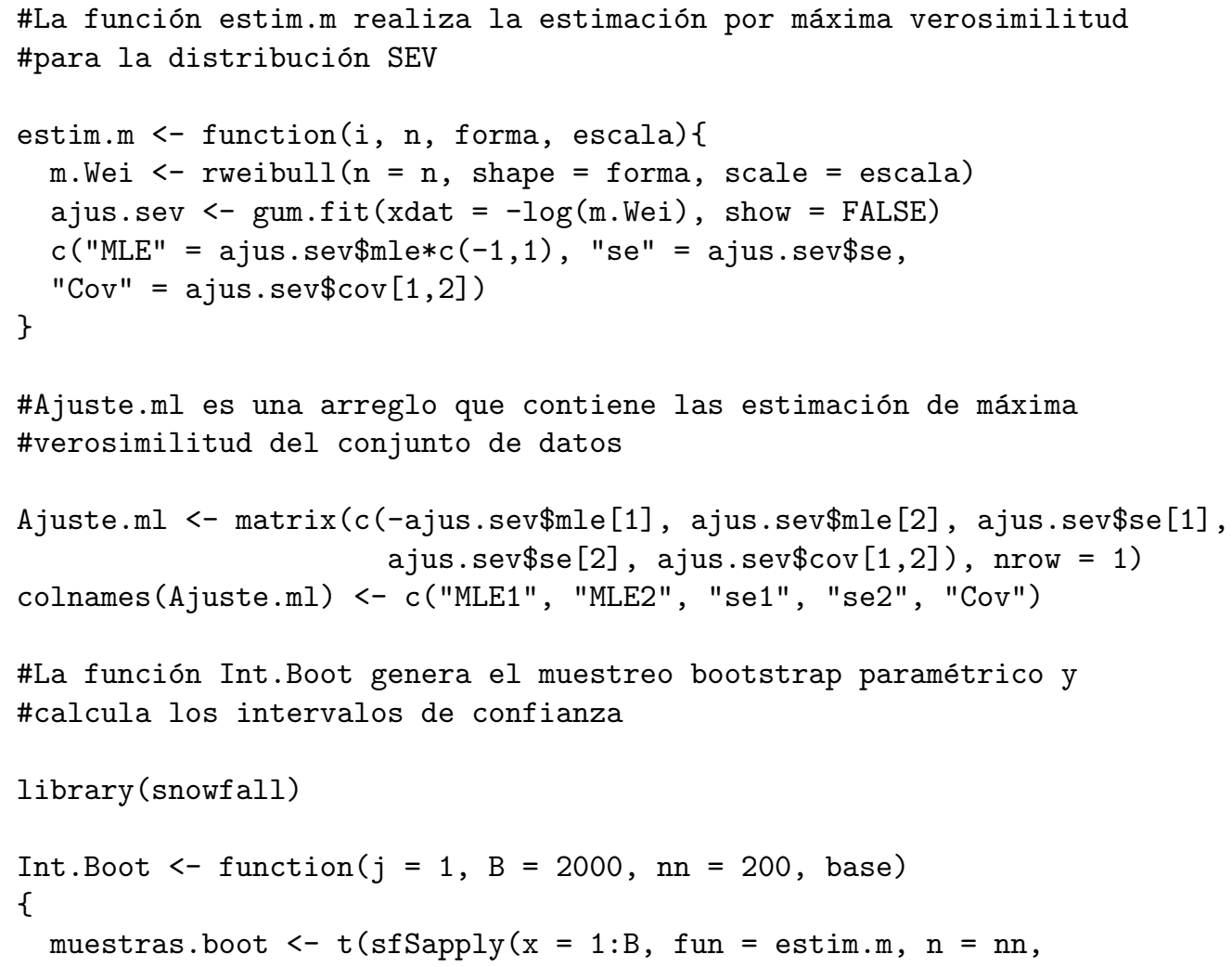




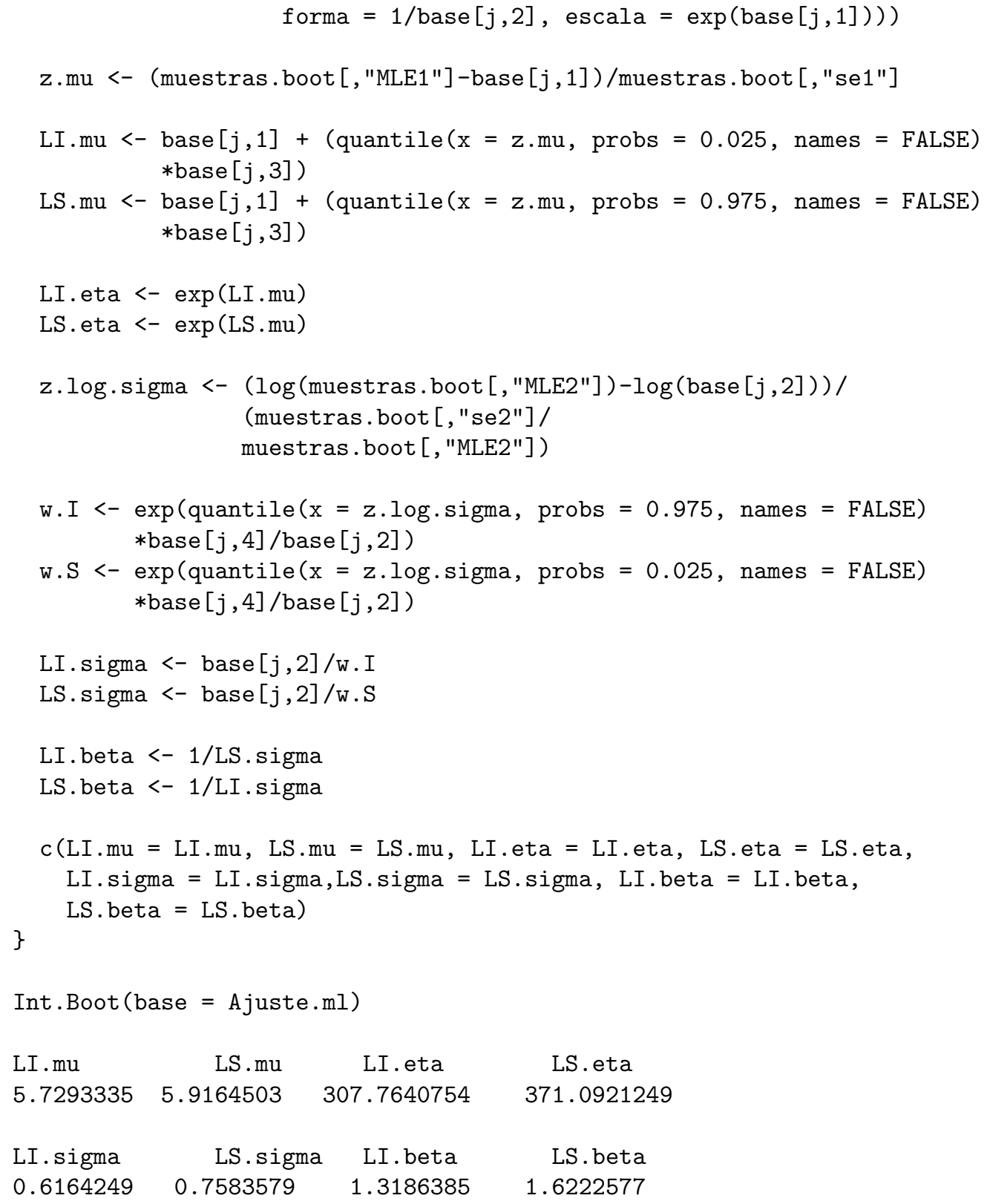

\section{Conclusiones}

Los intervalos de confianza estimados bajo aproximación normal para el parámetro de forma $\beta$ de la distribución Weibull resultaron asimétricos con respecto al error $\alpha$. 
En especial, cuando los tamaños de muestra son pequeños, $n=10$ o $n=20$, incluso en algunos casos estudiados, cuando $\beta=0.5$ no fue suficiente para corregir esta asimetría un tamaño de muestra $n=50$, lo que sugiere una lenta convergencia a la distribución normal de $\hat{\beta}=\frac{1}{\hat{\sigma}}$. Con respecto al comportamiento de las estimaciones de los límites del intervalo de confianza para el parámetro $\eta$ no se observa este tipo de comportamiento asimétrico de los intervalos obtenidos bajo aproximación normal.

Se recomienda hacer uso del método bootstrap paramétrico para la estimación por intervalos de los parámetros de escala $\eta$ y de forma $\beta$ de la distribución Weibull, puesto que en los escenarios simulados, se presentó un comportamiento deseable en lo referente a la estimación por intervalos de confianza: (a) intervalos de longitudes cercanas a las obtenidas por el método de aproximación normal (b) probabilidades de cobertura que alcanzan o superan la confianza nominal e (c) intervalos de confianza simétricos con respecto al error $\alpha$, aunque el límite superior del intervalo resultó altamente variable. La longitud de los intervalos obtenidos para el parámetro $\eta$ dependen de los valores del parámetro $\beta$ de la distribución Weibull, puesto que $\beta$ está estrechamente relacionado con el parámetro $\sigma$ de la SEV.

Recibido: 21 de febrero de $\mathbf{2 0 1 7}$ Aceptado: 9 de marzo de 2018

\section{Referencias}

Coles, S. (2001), An introduction to statistical modeling of extreme values, Springer.

Cousineau, D. (2009), 'Fitting the three-parameter weibull distribution: review and evaluation of existing and new methods', IEEE Transactions on Dielectrics and Electrical Insulations 16(1), 281-288.

Doganaksoy, N. (1995), Recent advances in life-testing and reliability, CRC Press, chapter Likelihood ratio confidence intervals in life data analysis, pp. 337-408.

Efron, B. (1985), 'Bootstrap confidence intervals for a class of parametric problems', Biometrika 72(1), 45-85.

Heffernan, J. E. \& Stephenson, A. G. (2016), ismev: An Introduction to Statistical Modeling of Extreme Values. R package version 1.41.

*https://CRAN.R-project.org/package=ismev

Jeng, S. \& Meeker, W. (2000), 'Comparisons of approximate confidence interval procedures for type i censored data.', Technometrics 42(2), 135-148.

Knaus, J. (2015), snowfall: Easier cluster computing (based on snow). R package version 1.84-6.1.

*https://CRAN.R-project.org/package=snowfall 
Koehler, E., Brown, E. \& Haneuse, S. (2009), 'On the assessment of monte carlo error in simulation-based statistical analyses', The American Statistician 63(2), 155-162.

Meeker, W. \& Escobar, L. (1998), Statistical Methods for Reliability Data, John Wiley and Sons, Inc.

Ostrouchov, G. \& Meeker, W. (1988), 'Accuracy of approximate confidence bounds computed from interval censored weibull and lognormal data', Journal of Statistical Computing and Simulations 29(1), 43-76.

Roth, T. (2016), qualityTools: Statistics in Quality Science. R package version 1.55 http://www.r-qualitytools.org.

*http://www.r-qualitytools.org

Team, R. C. (2017), R: A Language and Environment for Statistical Computing, R Foundation for Statistical Computing, Vienna, Austria.

*http://www.R-project.org/

Therneau, T. M. (2015), A Package for Survival Analysis in S. version 2.38. *https://CRAN.R-project.org/package=survival

Therneau, T. M. \& Grambsch, P. M. (2000), Modeling Survival Data: Extending the Cox Model, Springer, New York.

Vander Weil, S. \& Meeker, W. (1990), 'Accuracy of approximate confidence bounds using censored weibull regression data from accelerated life tests', IEEE Transactions on Reliability 39(6), 346-351. 


\section{A. Apéndice}

Algoritmo para la construcción de intervalos bootstrap paramétrico:

1. Considere $n$ observaciones $Y_{i}, i=1, \ldots, n$ con función de distribución $G_{\theta}(\cdot)$, donde $\theta$ puede ser un escalar o vector de parámetros de la distribución.

2. Obtenga una estimación MLE $\hat{\theta}$.

3. Defina el número de muestras boostrap $B$.

4. Genere $B$ muestras aletorias de $G_{\hat{\theta}}(y)$ con repetición y calcule el MLE $\hat{\theta}_{j}^{*}$, $j=1, \ldots, B$.

5. Calcule $Z_{\hat{\theta}_{j}^{*}}=\frac{\hat{\theta}_{j}^{*}-\hat{\theta}}{\hat{s e} \hat{\theta}_{j}^{*}}$ para $j=1, \ldots, B$, luego con los cuantiles $Z_{\hat{\theta}_{j,\left(1-\frac{\alpha}{2}\right)}^{*}}$, $Z_{\hat{\theta}_{j,\left(\frac{\alpha}{2}\right)}}$ construya el intervalo de confianza como en la expresión (3).
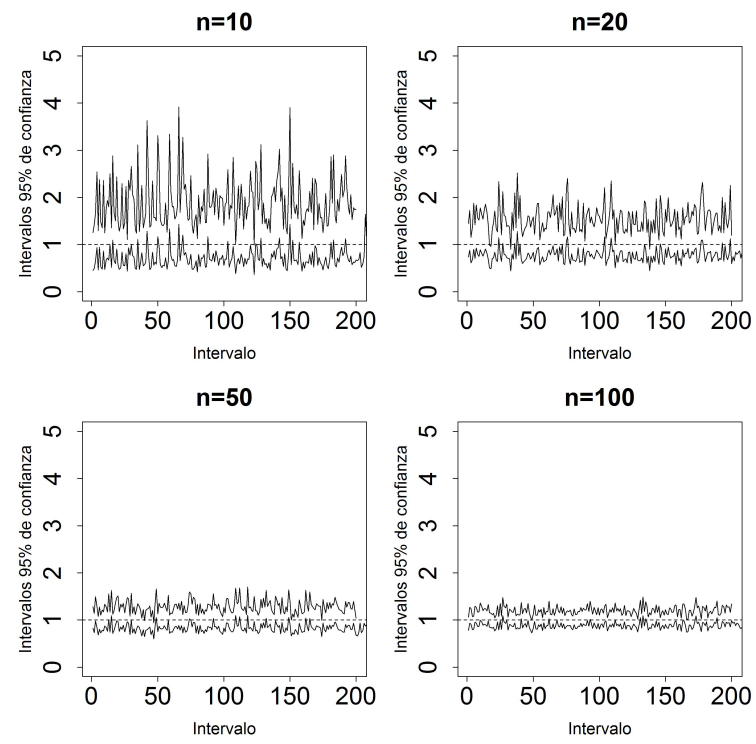

Figura 1: Comportamiento de la estimación de los limites de 200 Intervalos de confianza construidos a un $95 \%$ de confianza bajo aproximación normal para el parámetro de forma $\beta$, en el escenario de simulación $\beta=1, \eta=0.5$. La línea punteada indica el valor del parámetro, en este caso $\beta=1$ 
Tabla 1: Longitud mediana y probabilidad de cobertura para 10000 intervalos de confianza estimados para el parámetro de forma $\beta$ variando el parámetro $\eta$ y el tamaño de muestra.

\begin{tabular}{|c|c|c|c|c|c|c|c|c|}
\hline \multirow[b]{2}{*}{$n$} & \multirow[b]{2}{*}{$\beta$} & \multirow[b]{2}{*}{$\eta$} & \multicolumn{2}{|c|}{$\begin{array}{l}\text { Longitud } \\
\text { Mediana }\end{array}$} & \multicolumn{2}{|c|}{$\begin{array}{l}\text { Probabilidad } \\
\text { de cobertura }\end{array}$} & \multicolumn{2}{|c|}{ Indicadores } \\
\hline & & & $\mathrm{AN}$ & $\mathrm{BP}$ & $\mathrm{AN}$ & $\mathrm{BP}$ & RLMI & $\mathrm{RPC}$ \\
\hline \multirow[t]{3}{*}{10} & 0.5 & 0.5 & 0.56 & 0.53 & 0.91 & 0.96 & 1.06 & 0.95 \\
\hline & & 1 & 0.57 & 0.52 & 0.90 & 0.94 & 1.10 & 0.96 \\
\hline & & 2 & 0.57 & 0.53 & 0.92 & 0.96 & 1.08 & 0.96 \\
\hline \multirow[t]{3}{*}{10} & 1 & 0.5 & 1.14 & 1.06 & 0.91 & 0.95 & 1.08 & 0.96 \\
\hline & & 1 & 1.13 & 1.04 & 0.90 & 0.95 & 1.09 & 0.95 \\
\hline & & 2 & 1.13 & 1.04 & 0.90 & 0.94 & 1.09 & 0.96 \\
\hline \multirow[t]{3}{*}{10} & 2 & 0.5 & 2.26 & 2.07 & 0.91 & 0.95 & 1.09 & 0.96 \\
\hline & & 1 & 2.25 & 2.12 & 0.92 & 0.95 & 1.06 & 0.97 \\
\hline & & 2 & 2.27 & 2.14 & 0.91 & 0.95 & 1.06 & 0.96 \\
\hline \multirow[t]{3}{*}{20} & 0.5 & 0.5 & 0.34 & 0.37 & 0.93 & 0.94 & 0.92 & 0.99 \\
\hline & & 1 & 0.34 & 0.37 & 0.93 & 0.95 & 0.92 & 0.98 \\
\hline & & 2 & 0.40 & 0.37 & 0.93 & 0.95 & 1.08 & 0.98 \\
\hline \multirow[t]{3}{*}{20} & 1 & 0.5 & 0.74 & 0.74 & 0.93 & 0.96 & 1.00 & 0.97 \\
\hline & & 1 & 0.74 & 0.75 & 0.91 & 0.94 & 0.99 & 0.97 \\
\hline & & 2 & 0.74 & 0.73 & 0.93 & 0.95 & 1.01 & 0.98 \\
\hline \multirow[t]{3}{*}{20} & 2 & 0.5 & 1.48 & 1.46 & 0.92 & 0.96 & 1.01 & 0.96 \\
\hline & & 1 & 1.50 & 1.49 & 0.93 & 0.95 & 1.01 & 0.98 \\
\hline & & 2 & 1.47 & 1.46 & 0.93 & 0.96 & 1.01 & 0.97 \\
\hline \multirow[t]{3}{*}{50} & 0.5 & 0.5 & 0.22 & 0.22 & 0.95 & 0.95 & 1.00 & 1.00 \\
\hline & & 1 & 0.22 & 0.22 & 0.95 & 0.95 & 1.00 & 1.00 \\
\hline & & 2 & 0.22 & 0.22 & 0.93 & 0.95 & 1.00 & 0.98 \\
\hline \multirow[t]{3}{*}{50} & 1 & 0.5 & 0.44 & 0.44 & 0.94 & 0.94 & 1.00 & 1.00 \\
\hline & & 1 & 0.44 & 0.45 & 0.93 & 0.95 & 0.98 & 0.98 \\
\hline & & 2 & 0.45 & 0.44 & 0.94 & 0.95 & 1.02 & 0.99 \\
\hline \multirow[t]{3}{*}{50} & 2 & 0.5 & 0.89 & 0.89 & 0.94 & 0.95 & 1.00 & 0.99 \\
\hline & & 1 & 0.89 & 0.89 & 0.94 & 0.95 & 1.00 & 0.99 \\
\hline & & 2 & 0.89 & 0.89 & 0.95 & 0.96 & 1.00 & 0.99 \\
\hline \multirow[t]{3}{*}{100} & 0.5 & 0.5 & 0.15 & 0.15 & 0.95 & 0.95 & 1.00 & 1.00 \\
\hline & & 1 & 0.15 & 0.15 & 0.94 & 0.95 & 1.00 & 0.99 \\
\hline & & 2 & 0.15 & 0.15 & 0.95 & 0.95 & 1.00 & 1.00 \\
\hline \multirow[t]{3}{*}{100} & 1 & 0.5 & 0.31 & 0.31 & 0.95 & 0.95 & 1.00 & 1.00 \\
\hline & & 1 & 0.31 & 0.31 & 0.92 & 0.95 & 1.00 & 0.97 \\
\hline & & 2 & 0.31 & 0.31 & 0.94 & 0.95 & 1.00 & 0.99 \\
\hline \multirow[t]{3}{*}{100} & 2 & 0.5 & 0.62 & 0.62 & 0.95 & 0.96 & 1.00 & 0.99 \\
\hline & & 1 & 0.63 & 0.62 & 0.94 & 0.94 & 1.02 & 1.00 \\
\hline & & 2 & 0.62 & 0.62 & 0.95 & 0.94 & 1.00 & 1.01 \\
\hline
\end{tabular}

Comunicaciones en Estadística, junio 2018, Vol. 11, No. 1 
Tabla 2: Longitud mediana y probabilidad de cobertura para 10000 intervalos de confianza estimados para el parámetro de escala $\eta$ variando el parámetro $\beta$ y el tamaño de muestra.

\begin{tabular}{|c|c|c|c|c|c|c|c|c|}
\hline \multirow[b]{2}{*}{$n$} & \multirow[b]{2}{*}{$\eta$} & \multirow[b]{2}{*}{$\beta$} & \multicolumn{2}{|c|}{$\begin{array}{l}\text { Longitud } \\
\text { Mediana }\end{array}$} & \multicolumn{2}{|c|}{$\begin{array}{l}\text { Probabilidad } \\
\text { de cobertura }\end{array}$} & \multicolumn{2}{|c|}{ Indicadores } \\
\hline & & & $\mathrm{AN}$ & $\mathrm{BP}$ & $\mathrm{AN}$ & $\mathrm{BP}$ & RLMI & $\mathrm{RPC}$ \\
\hline \multirow[t]{3}{*}{10} & 0.5 & 0.5 & 1.35 & 1.96 & 0.90 & 0.94 & 0.69 & 0.96 \\
\hline & & 1 & 0.63 & 0.77 & 0.90 & 0.95 & 0.82 & 0.95 \\
\hline & & 2 & 0.30 & 0.37 & 0.89 & 0.95 & 0.81 & 0.94 \\
\hline \multirow[t]{3}{*}{10} & 1 & 0.5 & 3.10 & 4.02 & 0.90 & 0.95 & 0.77 & 0.95 \\
\hline & & 1 & 1.23 & 1.57 & 0.89 & 0.95 & 0.78 & 0.94 \\
\hline & & 2 & 0.60 & 0.72 & 0.89 & 0.96 & 0.83 & 0.93 \\
\hline \multirow[t]{3}{*}{10} & 2 & 0.5 & 5.76 & 7.72 & 0.89 & 0.95 & 0.75 & 0.94 \\
\hline & & 1 & 2.38 & 3.13 & 0.89 & 0.95 & 0.76 & 0.94 \\
\hline & & 2 & 1.17 & 1.47 & 0.89 & 0.95 & 0.80 & 0.94 \\
\hline \multirow[t]{3}{*}{20} & 0.5 & 0.5 & 0.97 & 1.11 & 0.93 & 0.94 & 0.87 & 0.99 \\
\hline & & 1 & 0.45 & 0.49 & 0.93 & 0.94 & 0.92 & 0.99 \\
\hline & & 2 & 0.22 & 0.24 & 0.92 & 0.94 & 0.92 & 0.98 \\
\hline \multirow[t]{3}{*}{20} & 1 & 0.5 & 1.99 & 2.12 & 0.95 & 0.95 & 0.94 & 1.00 \\
\hline & & 1 & 0.90 & 0.98 & 0.92 & 0.93 & 0.92 & 0.99 \\
\hline & & 2 & 0.44 & 0.49 & 0.93 & 0.95 & 0.90 & 0.98 \\
\hline \multirow[t]{3}{*}{20} & 2 & 0.5 & 3.89 & 4.41 & 0.93 & 0.96 & 0.88 & 0.97 \\
\hline & & 1 & 1.78 & 1.99 & 0.93 & 0.95 & 0.89 & 0.98 \\
\hline & & 2 & 0.89 & 0.98 & 0.92 & 0.93 & 0.91 & 0.99 \\
\hline \multirow[t]{3}{*}{50} & 0.5 & 0.5 & 0.59 & 0.61 & 0.92 & 0.95 & 0.97 & 0.97 \\
\hline & & 1 & 0.29 & 0.30 & 0.94 & 0.94 & 0.97 & 1.00 \\
\hline & & 2 & 0.14 & 0.15 & 0.94 & 0.95 & 0.93 & 0.99 \\
\hline \multirow[t]{3}{*}{50} & 1 & 0.5 & 1.20 & 1.28 & 0.94 & 0.94 & 0.94 & 1.00 \\
\hline & & 1 & 0.58 & 0.59 & 0.95 & 0.94 & 0.98 & 1.01 \\
\hline & & 2 & 0.29 & 0.30 & 0.95 & 0.95 & 0.97 & 1.00 \\
\hline \multirow[t]{3}{*}{50} & 2 & 0.5 & 2.41 & 2.51 & 0.93 & 0.95 & 0.96 & 0.98 \\
\hline & & 1 & 1.16 & 1.20 & 0.95 & 0.95 & 0.97 & 1.00 \\
\hline & & 2 & 0.57 & 0.60 & 0.94 & 0.95 & 0.95 & 0.99 \\
\hline \multirow[t]{3}{*}{100} & 0.5 & 0.5 & 0.42 & 0.42 & 0.95 & 0.95 & 1.00 & 1.00 \\
\hline & & 1 & 0.21 & 0.21 & 0.94 & 0.94 & 1.00 & 1.00 \\
\hline & & 2 & 0.10 & 0.10 & 0.93 & 0.95 & 1.00 & 0.98 \\
\hline \multirow[t]{3}{*}{100} & 1 & 0.5 & 0.84 & 0.85 & 0.94 & 0.96 & 0.99 & 0.98 \\
\hline & & 1 & 0.41 & 0.42 & 0.94 & 0.95 & 0.98 & 0.99 \\
\hline & & 2 & 0.20 & 0.21 & 0.95 & 0.96 & 0.95 & 0.99 \\
\hline \multirow[t]{3}{*}{100} & 2 & 0.5 & 1.67 & 1.69 & 0.95 & 0.94 & 0.99 & 1.01 \\
\hline & & 1 & 0.82 & 0.84 & 0.94 & 0.94 & 0.98 & 1.00 \\
\hline & & 2 & 0.41 & 0.41 & 0.95 & 0.95 & 1.00 & 1.00 \\
\hline
\end{tabular}



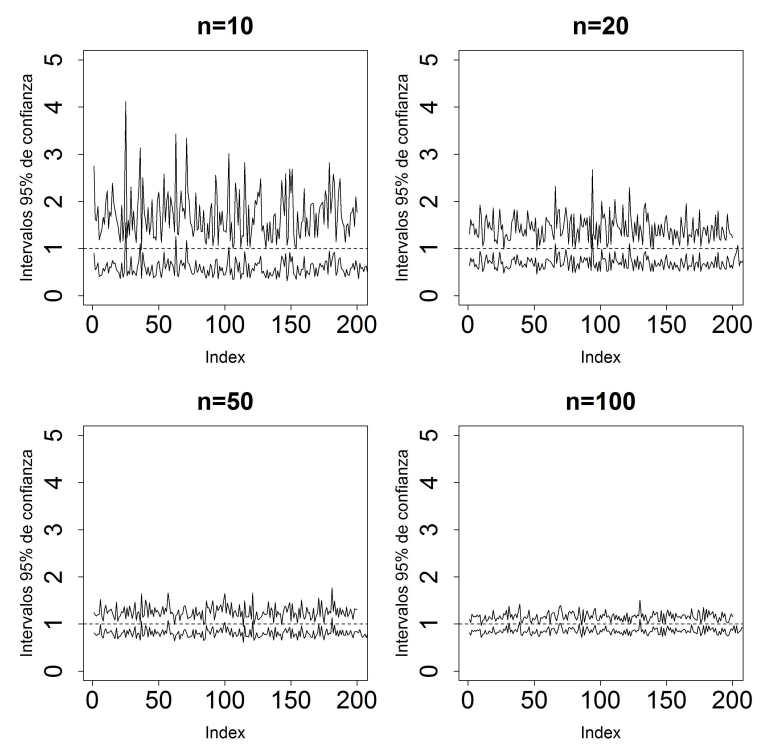

Figura 2: Comportamiento de la estimación de los limites de 200 Intervalos de confianza construidos a un $95 \%$ de confianza bajo bootstrap para el parámetro de forma $\beta$, en el escenario de simulación $\beta=1, \eta=0.5$. La línea punteada indica el valor del parámetro, en este caso $\beta=1$ 

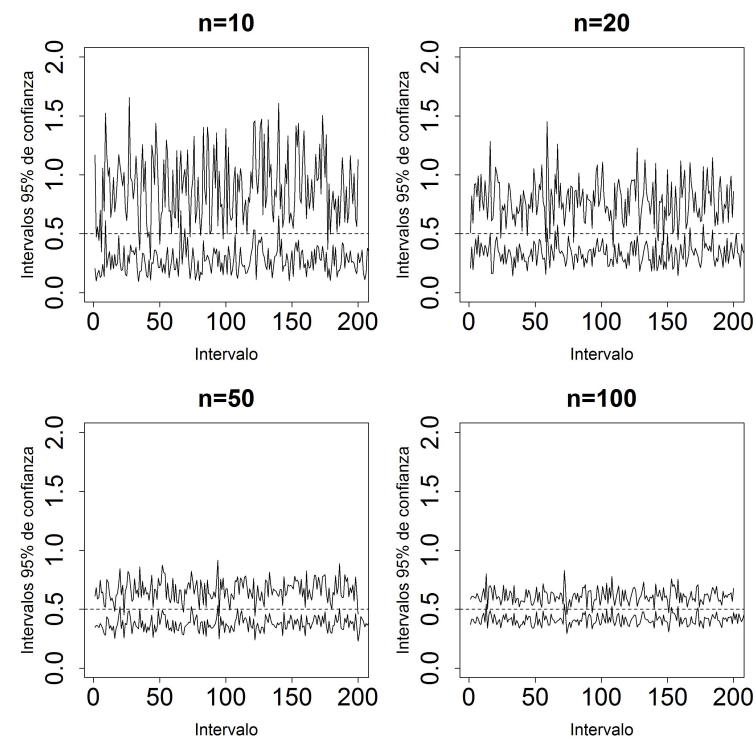

Figura 3: Comportamiento de la estimación de los limites de 200 Intervalos de confianza construidos a un $95 \%$ de confianza bajo aproximación normal para el parámetro de escala $\eta$, en el escenario de simulación $\beta=1, \eta=0.5$. La línea punteada indica el valor del parámetro, en este caso $\eta=0.5$ 

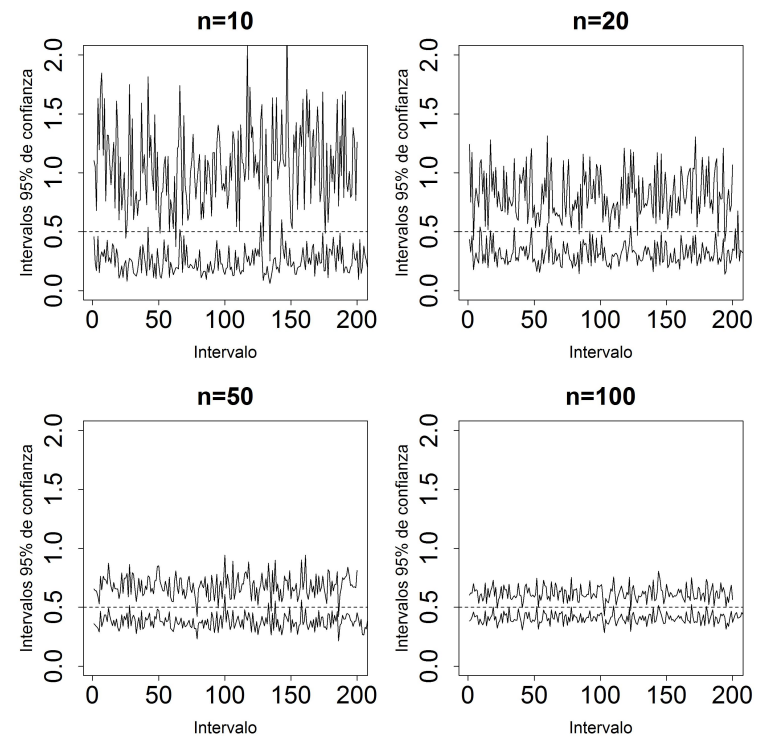

Figura 4: Comportamiento de la estimación de los limites de 200 Intervalos de confianza construidos a un $95 \%$ de confianza bajo bootstrap para el parámetro de escala $\eta$, en el escenario de simulación $\beta=1, \eta=0.5$. La línea punteada indica el valor del parámetro, en este caso $\eta=0.5$

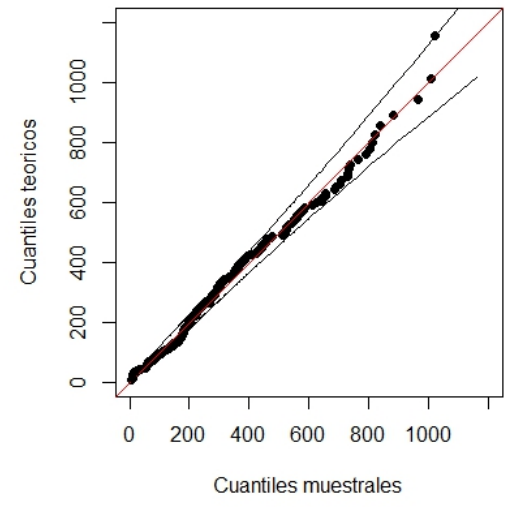

Figura 5: Gráfico cuantil-cuantil para la distribución Weibull 